\title{
Genetics of Cherubism
}

\author{
Nikita Syahrussiami Firdaus ${ }^{1}$, Elza Ibrahim Auerkari ${ }^{2 *}$ \\ ${ }^{1}$ Pediatric Dentistry Residency Program, Faculty of Dentistry, University of Indonesia, Jakarta \\ 10430, Indonesia \\ ${ }^{2}$ Department of Oral Biology, Faculty of Dentistry, University of Indonesia, Jakarta 10430, \\ Indonesia \\ *Email: elza.ibrahim@ui.ac.id
}

\begin{abstract}
Cherubism, characterized by bone resorption limited to either side of the jaws, is a rare bone dysplasia that leads to facial swelling. The swelling is symmetrical, hard, and painless. The disorder typically begins in childhood and then slowly decelerates, stopping at puberty. Studies have reported the existence of both mild and severe cases of cherubism. It can be caused by various types of genetic mutation. Most of them are point mutations and can be transitions, translations, or deletions. The type of mutation will determine whether the condition is mild or severe. It is important to know and understand the genetic factors of cherubism to determine the correct diagnosis and treatment plan for the disease. In this paper, we will discuss the etiology, manifestation, pathophysiology, and genetics of cherubism.
\end{abstract}

Keywords: cherubism, genetics, SH3BP2 gene, point mutation

\section{Introduction}

Cherubism is a rare, hereditary disease that begins in childhood and is characterized by proliferative lesions of fibrous tissue and swelling of the lower third part of the face. Affected children have an appearance resembling the look of cherubs that appear in Renaissance-era paintings, due to a large number of giant cells within the mandible and maxilla [1,2]. Soft, filamentous tissue rich in giant cells can widen and cause facial malformation as it fills bone lesions. Bone resorption limited to either side of the jaws results in a rare bone dysplasia [3]. It affects all four quadrants of the jaw, causing a characteristic round and symmetrically full lower face in most patients [2]. Symmetrical, hard, and pain-free enlargement of the jaws is exhibited by patients with cherubism. The lower part of the eyelid is pulled down and extends the skin of the cheeks. Subjection of the inferior part of the sclerae is the result of the eyeballs slanting upward, which is due to the involvement of the infraorbital rim and the orbital floor $[1,4]$.

Cherubism is inherited as an autosomal dominant disorder with a higher frequency in boys than in girls. The ratio of affected boys to affected girls is $2: 1.2,5$ Jones in 1933 was the first person to depict cherubism as "familial multilocular cystic lesion of the jaw" [2]. In Jones' 1950 review, for the malformation that he had first characterized 17 years earlier, a multilocular cystic disease of the jaws, Jones suggested the name "cherubism" [3]. Symptoms usually begin to appear between the ages of 2 and 5 years. Bone resorption and enlargement of the face continue until the children reach puberty, at which time symptoms stabilize and slowly regress [2,3]. The gene responsible for cherubism was discovered by recent 
genetic research. The mutation is detected in a gene called SH3BP2, which is localized to chromosome $4 \mathrm{p} 16.3$. The existence of multiple mutations and incomplete penetrance are the causes of variability in the appearance or clinical manifestations of cherubism $[2,5]$.

\section{Cherubism}

\subsection{Definition}

In 1933, William A. Jones was the first person to independently suggest that cherubism was a multilocular, cystic disease upon learning of several affected members of the same family. Due to the resemblance of the clinical manifestations of the disease to a cherubic style of drawing that was popular in the Renaissance era, he decided to name the condition cherubism. This was adopted as the conventional nomenclature because the name precisely described the clinical appearance and features of cherubism $[4,6,7]$.

Cherubism makes its first appearance at the age of 2 to 7 years, usually with clinical manifestations such as symmetrical, expansive, multilocular, radiolucent lesions on both sides of the jaws, in the maxilla and/or mandible. Following the appearance of the first symptoms and diagnosis of the condition, manifestations of the lesions increase for the first two years and begin to stabilize or even regress to a stop when the child reaches puberty. Pain-free, cyst-like structures develop and substitute for bone as the upper and lower jaws expand [5-7,8].

\subsection{Classification}

A clinical classification system has been recommended to explain the various levels of severity and locations of the lesions in cherubism. The grading system can be seen on the table below. Grade 1: Lesions of the mandible that do not involve root resorption. Grade 2: Lesions involving the mandible and maxilla without signs of root resorption. Grade 3: Aggressive lesions of the mandible with signs of root resorption. Grade 4: Lesions involving the mandible and maxilla and showing signs of root resorption. Grade 5: Rare, massively growing, aggressive, and extensive deformity caused by juvenile lesions involving the mandible and maxilla, which may include the coronoid and condyles. Grade 6: Rare, massively growing, aggressive, and extensively deforming juvenile cases involving the maxilla, mandible, and orbits. The first grading system was proposed by Raposo-Amaral and later simplified by Motamedi, who added grade 6 to explain the involvement of the orbits [6]. PerezSayans et al. modified the clinical classification or grading system for cherubism once again in 2017 . They added grade 0 to include cases in which a mutation exists, but the disease manifestation is not expressed [9].

\subsection{Etiopathogenesis}

At least 11 mutations in the SH3BP2 gene have been discovered in patients with cherubism. A frameshift mutation occurs in a critical region of the SH3BP2 gene, resulting in a very active form of the protein. Although the outcomes of this mutation are as yet unknown, they are assumed to be very important. Many authors believe that the mutated protein causes a shift in crucial signaling pathways, as specific 
immune system cells and the preservation of bone tissue are related to cellular signaling [7].

Intensified osteoclast generation could be the result of stimulation by an overly active protein in the jaw bones. Abnormal destruction of the bone tissue in the maxilla and mandible is assisted by a surplus of osteoclasts. The cyst-like development traits of cherubism are caused by a mixture of bone resorption and infection. The osteoclastic lesions of the bone in cherubism could be caused by intensified tartrateresistant acid phosphate and nuclear factor of activated T-cells, which are also results of the mutation. Studies indicate that SH3BP2 functions in the regulation of bone homeostasis by osteoclast-specific effects and through effects on differentiation and function of osteoblasts [7].

\subsection{Role of genes in cherubism}

Cherubism is a rare disease with an autosomal dominant pattern of inheritance linked to the SH3BP2 gene. Some studies suggest the existence of a recessive form of the disease. In some patients, genetic examination revealed no mutations, although the patient had been diagnosed with cherubism. This raises the possibility of genetic heterogeneity in SH3BP2. Presumed to be new mutations with no family history, approximately half of the cases of cherubism associated with genetic mutations were identified at the University of Connecticut Health Center $[3,6]$.

A thorough statistical analysis revealed a significant difference in penetrance between males and females. Variable expressivity within families has been reported, with data suggesting $100 \%$ penetrance in males and a reduction to $70 \%-50 \%$ in females. Some female patients began developing symptoms or clinical manifestations of cherubism after a checkup was already performed, which may be one of the reasons why penetrance in females appears to be lower than in males. Some studies said that symptoms of cherubism may no longer be apparent on the radiographs of older patients. We should bear in mind that bone lesions in some more mature patients with a mild form of cherubism could have been reorganized into a normal mandibular bone before the examination was conducted [3]. In addition to the known variable penetrance and expressivity, irregular cases of cherubism have been noted [10].

There have been several reported cases of familial cherubism. One example is a case presented by Perez-Sayans et al., in which they studied the variable expressivity of familial cherubism. Because of a serious malposition of the teeth, a 37-yearold male patient who had been diagnosed with cherubism when he was 9 years old came to a maxillofacial surgery department. The man had one brother and one sister with no signs of cherubism. The sister had two children. The older one was diagnosed with cherubism, whereas the younger one did not inherit the mutation and presented a normal genotype. Affected female members of a family were also reported in other cases. Li et al. reported on a household in Northern China in which all 21 residents were relatives and three of the female family members were diagnosed with cherubism [10,11]. Most of the mutations associated with cherubism have been identified as point mutations in the SH3BP2 gene, which is localized to chromosome $4 \mathrm{p} 16.3$ [12]. 


\subsection{The SH3BP2 gene and its function}

The gene responsible for cherubism was discovered in a recent genetic study. $\mathrm{SH} 3$ binding protein 2 (SH3BP2) is a 48-kbp gene located on chromosome 4p16.3 [2,6] and is composed of 13 exons encoding a 561-amino-acid, 62-kDa protein. Three modular peptide recognition domain structures are found within the SH3BP2 gene, which also consists of a proline-rich or PR domain, referred to by some as a 10amino-acid SH3 binding site. The protein also contains an N-terminal pleckstrin homology or PH domain and a C-terminal SH2 domain, which is identical to the Src homology 2 domain found in most adapter proteins [2,3].

First recognized in humans and mice, the adapter protein SH3BP2 is a protein tyrosine kinase $\mathrm{c}$ Abl-binding protein. Most human and mice proteins share some amino acid sequence identity. The SH3BP2 homolog in humans was predicted to share $87 \%$ amino acid sequence similarity with the mouse protein, and approximately $81 \%$ DNA sequence homology was identified in the coding region. Using its $\mathrm{SH} 3$ binding domain, the protein can also bind to c Abl. The product of the SH3BP2 gene is expressed in almost all cell types. SH3BP2, which serves as an adapter protein, regulates intracellular signaling by establishing complexes with scaffolding and binding proteins. According to another study, the normal physiological role of the SH3BP2 gene is to encode a protein of as yet unknown function, which is also notable for communication with other proteins. In bone remodeling (replacement and reorganization of old bone tissue), the SH3BP2 protein transmits cellular signals to the immune system cells that carry out this process. In patients suffering from Wolf-Hirschhorn syndrome, the SH3BP2 gene is located in a zone that is very often deleted, resulting in haploinsufficientcy of SH3BP2, but cherubism or cherubism-like clinical manifestations are not produced in these individuals $[3,13,14]$.

Using the $\mathrm{SH} 3$ binding motif in the proline-rich domain, SH3BP2 is speculated to bind to cell membrane lipids using its $\mathrm{PH}$ domain to communicate with the $\mathrm{SH} 3$ binding partners. Carrying a Tyr Glu Asn binding motif, the SH2 domain can interact with a number of binding partners [3].

$\mathrm{SH} 3 \mathrm{BP} 2$ is an adaptor protein, mostly acting on immune cells such mast cells, $\mathrm{T}$ cells, neutrophils, B cells, and macrophages as well as osteoclasts. It arranges intracellular signaling pathways in skeletal and immune systems and also communicates with various protein such as VAV, SRC, SYK, and PLCr. A previous study reported that the human craniofacial disorder known as cherubism, which manifests as excessive jawbone destruction with enlargement of the lower third part of the face, is caused by gain-of-function mutations in the SH3BP2 gene. Increased osteoclastogenesis, which has been indicated as the cause of massive jawbone resorption, suggested that jaw lesions are composed of a large number of tartrate- resistant acid phosphate positive multinucleated giant cells with fibroblastoid cells in general $[15,16]$.

\subsection{Genetic pathogenesis}

Exactly how mutations in SH3BP2 lead to cherubism is not yet known, but they may act by interfering with cellular signaling. NFAT (nuclear factor of activated T 
cells) is activated by SH3BP2, which is an important component of signaling pathways and could bind to the cytosolic proteins SYK, Lat, and 14-3-3.

A recent study showed that the mutation increased NFAT activity [3]. NFAT has been suggested as the master transcription factor in osteoclastogenesis. Experimental evidence supports a critical role for the protein in osteoclast development. Some authors believe that the mutations found in patients with cherubism reinforce the involvement of signaling pathways in osteoclast development and activation [2].

According to recent study in a mouse model by Ueki et al., the SH3BP2 mutation causes increased myeloid cell responses to M-CSF and RANKL stimulation, and these cells take the shape of large osteoclasts and macrophages that express TNF- $\alpha$ at a high level. Based on these findings and those of others, we suggest that multinucleated giant cells in the lesion may be formed from monocyte/macrophage precursors. Under the influence of RANKL expressed in stromal cells, the precursor cells may differentiate into osteoclasts; therefore, they are believed to be osteoclastic in nature [2].

\subsection{SH3BP2 gene mutation}

A study by Ueki et al. (2001) demonstrated point mutations in the SH3BP2 gene in 12-15 families. All of these point mutations were identified in exon 9 and resulted in amino acid substitutions within a 6-amino acid sequence from position 415 to 420 in the protein (RSPPDG) in the proline-rich domain proximal to the $\mathrm{SH} 2$ domain of SH3BP2 [2,3]. A recent study revealed no SH3BP2 gene mutations in patients with aggressive central giant cell granuloma. These results indicate that a single genetic factor is responsible for cherubism, and we can use the results of this genetic analysis for specific diagnosis of cherubism [2,3].

The mutation described by Lee et al. (Pro418Arg) is in a well-known mutational hotspot at amino acid numbers 415-420, allowing us to distinguish between cherubism and central giant cell granuloma. According to the authors, this is the second SH3BP2 gene mutation identified in association with cherubism in Asian populations. Though both cases in the study had this point mutation, the patient in this case showed clinical features of cherubism, although his mother did not show the phenotype. The reason we assumed a diagnosis of cherubism is that, as stated earlier, the penetrance of cherubism is higher in men $(100 \%)$ than in women $(50 \%-70 \%)$ [2].

Another study that cited Ueki et al. stated that cherubism may even occur in those without a family history of cherubism. Based on this report, the researchers focused on the fact that cherubism, which is an autosomal dominant characteristic that can be inherited, can be easily diagnosed by DNA genetic analysis using leukocytes obtained from blood collection. The article reported DNA sequencing of the PCR amplification product of the SH3BP2 gene in exon 9, which indicated a heterozygous missense mutation at codon 415 (CGA $>$ CAA), in the patient and his mother, resulting in an amino acid change from Arg to Gln. The mutation was not present in the father.

\subsection{Point mutations in the SH3BP2 gene}

Mutations can be point alterations involving only a single nucleotide pair in the sequence, or they can be changes in larger sections of DNA at the chromosome 
level. An addition, deletion, or alteration of a single base pair can commonly and directly affect the function of only one gene and is called a point mutation [17].

A point mutation can be a base pair substitution, which maintains the reading frame of the codon sequence. It can also be an addition or deletion, which shifts the entire reading frame (frameshift mutation). There are two types of single base pair substitution mutations: transversions and transitions. A transversion replaces a purine with a pyrimidine or a pyrimidine with a purine, whereas a transversion replaces one pyrimidine for another or replaces a purine with another purine. The nature of the substitution and its location are the two factors that control the consequences of a substitution mutation in the protein coding region of a gene. A point mutation that does not change the amino acid sequence of the protein is known as a silent mutation $[17,18]$.

A mutation that results in the exchange of one amino acid for another in the protein sequence is a called a missense mutation. When a change in a nucleotide base inserts a stop codon, which results in premature translation termination and a truncated protein, it is called a nonsense mutation. Addition or deletion of a number of nucleotides that is not a multiple of three alters the reading frame; this is called a frameshift mutation. A frameshift mutation commonly results in a premature stop codon downstream of the insertion or deletion site [17].

Substitutions of the amino acids Pro 418, Gly 420, and Arg 415 are each the result of a single point mutation in the SH3BP2 gene. These mutations account for all of those detected in the laboratory of UCHC. In two Brazilian cherubism patients, an exceptional mutation in the domain of pleckstrin homology was found by Carvalho et al. A Thr 107 Met substitution was found in tumor tissue and in the germline or blood, the result of a point mutation in exon 4 [3].

A frameshift of more than 26 amino acids, which resulted a stop at location 325 (p.Arg49ArgfsX26) or a premature stop codon was found in the tumor tissue of another patient. A deletion of a single nucleotide at position 147 (c.147delC) existed in the same group but had another variant. To the best of the researchers' knowledge, this was the only patient who suffered from a lethal form of cherubism. The truncated protein (the N-terminal 48 amino acids) resulted in a rapidly progressing and fatal form of cherubism [3].

A hostile demeanor in a patient with cherubism was reported in another study by Carvalho et al., which revealed a novel mutation. It appeared to be a deletion of a single nucleotide located at position 408 (408delC). The deletion occurred in a region of the gene that encodes an N-terminal domain with significant pleckstrin homology. Membrane trafficking and cell adhesion are examples of biological functions mediated by the PI3 kinase effector that act through its PH domain. Strong lipid binding specificity was displayed by only a few $\mathrm{PH}$ domains, targeting proteins to the plasma membrane. How SH3BP2 mutations could have led to phenotypic variation in cherubism is still undetermined, even though mutations affected varying protein domains, according to almost every study [13].

The Noonan spectrum of phenotypes, which includes multiple giant lesions or Noonan-like syndrome, is a disease in which multilocular cysts occur, resembling 
cherubism. Craniofacial malformations, short stature, cardiac abnormalities, cryptorchidism, and webbed neck are a few examples of clinical manifestations of Noonan syndrome [19, 20].

To date, there has only been one report of central giant cell lesions resulting from a somatic mutation in the SH3BP2 gene. The mutation occurred in the $\mathrm{SH} 2$ domain of SH3BP2 where a point mutation in exon 11 results in substitution of glutamine 481 to leucine [21].

\subsection{Epigenetics}

Epigenetics, a comparatively new area of molecular biology, deals with heritable alterations in gene activity which are not the result of changes in the nucleotide sequence of DNA. According to recent studies, shifts in gene expression during differentiation are passed on to daughter cells through mitosis and can be inherited by subsequent generations. Neither of the two cases above are related to changes in the sequence of DNA; therefore, both of them are considered as epigenetic mechanisms. In the first case, individual genes are transmitted by cells of germ line according to a hereditary mechanism. In the second case, gene expression during growth is altered by mechanism that can be inherited [22].

Reciprocal action between the environment and the complex process of growth in an organism is one way to describe the meaning of epigenetics. It is vital that the normal alterations and growth of particular cell ancestry be maintained in the mature organism. Being involved in the process of alteration, epigenetic programing has a vital role in the organization of pluripotency genes [23].

DNA methylation is the best-known epigenetic mechanism. For example, the state of gene expression is transmitted from parent to daughter cells via DNA methylation. Methylation at specific cytosine positions in DNA is implicated in the recognition of epigenetic markers. Because of de novo methylation, at the start of embryogenesis in mammals, the degree of DNA methylation declines greatly.

DNA methylation patterns are transmitted by epigenetic inheritance. It involves the introduction of epigenetic markers by methylation at specific positions on cytosine in DNA (addition of methyl group into the carbon-5 position in the cytosine ring). The level of DNA methylation decreases sharply during early embryogenesis in mammals but later recovers due to de novo methylation. Methylation is vital for regulation of embryonic stem cell genes, and undifferentiated stem cell can be methylated. CpG methylation plays a role in two important mechanisms. The first one is $\mathrm{X}$ chromosome inactivation and genomic imprinting. The second one is the guaranteed repression of transcription of transposons and repeat elements. The expression of some tissue-specific genes is regulated during differentiation and development by repression of their expression by transcriptional silencing gene via $\mathrm{CpG}$ methylation $[22,24]$.

Histone modification is vital to the functional and structural organization of chromatin. It can influence many processes related to DNA, for example, chromosome organization, transcription, DNA replication and repair, and recombination. The difference between DNA methylation and histone modification lies in the dynamics. Histone modification is more dynamic and difficult to analyze while DNA methylation is more stable. 
Mutations in the SH3BP2 gene may interfere with signaling. NFAT is activated by an important part of a signaling pathway in which various cytosolic proteins can bind to SH3BP2. Recent studies reported intensification of NFAT action by SH3BP2 mutation. A crucial role of the protein in osteoclast development is supported by experimental evidence, and NFAT has been suggested as the transcriptional regulatory factor for osteoclastogenesis. The signaling pathways that guide the formation and growth of the osteoclast might be reinforced by mutation as the authors suggest. 2 According to a recent study by Ueki et al. in a mouse model of cherubism, myeloid cells respond to RANKL and M-CSF input is increased because of the mutation in SH3BP2. RANKL expression in stromal cells may cause precursor cell differentiation to osteoclasts. For this reason, they are well known to be osteoclastic. Alterations in the state of chromatin are vital for osteoclastogenesis, which is induced by RANKL, as recent evidence indicates. Sadly, osteoclasts have not been targeted therapeutically and are not well understood regarding epigenetic mechanisms [25].

\section{Conclusion}

Cherubism is a rare disease attributed to mutation in the SH3BP2 gene. Variable expressivity within families has been reported, with data suggesting $100 \%$ penetrance in males decreasing to $70 \%-50 \%$ in females. The gene responsible for cherubism has been discovered in a recent genetic study, namely SH3 binding protein 2 and located on chromosome 4p16.3. Mutations identified in exon 9 resulted in amino acid substitutions within a 6-amino-acid sequence from position 415 to 420. The activity of NFAT, the transcription factor which controls osteoclastogenesis, is increased by SH3BP2 mutation. RANKL-induced alterations in chromatin are important for osteoclastogenesis. Sadly, osteoclasts have not been targeted therapeutically and are not well understood regarding epigenetic mechanisms.

\section{References}

1. Mangion J, Rahman N, Edkins S, Barfoot R, Nguyen T, Sigurdsson A, et al. The gene for cherubism maps to chromosome 4p16. 3. Am J Hum Genet. 1999;65(1):151-7.

2. Lee JY, Jung YS, Kim SA, Lee SH, Ahn SG, Yoon JH. Investigation of the SH3BP2 gene mutation in cherubism. Acta Med Okayama. 2008 Jun;62(3):209-12.

3. Reichenberger EJ, Levine MA, Olsen BR, Papadaki ME, Lietman SA. The role of SH3BP2 in the pathophysiology of cherubism. Orphanet J Rare Dis. 2012;7(1):S5.

4. Tiziani V, Reichenberger E, Buzzo CL, Niazi S, Fukai N, Stiller M, et al. The gene for cherubism maps to chromosome 4p16. Am J Hum Genet. 1999 Jul; 65(1): 158-166.

5. Lima GD, Almeida JD, Cabral LA. Cherubism: clinicoradiographic features and treatment. J Oral Maxillofac Res. 2010 Apr-Jun; 1(2): e2.

6. Papadaki ME, Lietman SA, Levine MA, Olsen BR, Kaban LB, Reichenberger EJ. Cherubism: best clinical practice. Orphanet J Rare Dis. 2012 Jun;7(1):. S6.

7. Rageh OA. Parameters for Cherubism diagnosis: Clinicoradiographic features, Biochemical, Cytogenetic, Immunohistochemistry, and histopathology. J Dent Med Sci. 2016;15(10):86-91.

8. Mukherjee PM, Wang CJ, Chen IP, Jafarov T, Olsen BR, Ueki Y, Reichenberger EJ. Cherubism gene Sh3bp2 is important for optimal bone formation, osteoblast differentiation, and function. Am J Orthod Dentofac. 2010;138(2):140-e1. 
9. Jiao Y, Zhou M, Yang Y, Zhou J, Duan X. Cherubism misdiagnosed as giant cell tumor: a case report and review of literature. Int J Clin Exp Med. 2015;8(3):4656.

10. Pérez-Sayáns M, Barros-Angueira F, Suárez-Peñaranda JM, García-García A. Variable expressivity familial cherubism: woman transmitting cherubism without suffering the disease. Head Face Med. 2013;9(1):33.

11. Li CY, Yu SF. A novel mutation in the SH3BP2 gene causes cherubism: case report. BMC Med Genet. 2006 Dec;7(1):84.

12. Carvalho VM, Perdigão PF, Pimenta FJ, de Souza PE, Gomez RS, De Marco L. A novel mutation of the SH3BP2 gene in an aggressive case of cherubism. Oral Oncol. 2008;44(2):153-5.

13. Carvalho VM, Perdigao PF, Amaral FR, De Souza PE, De Marco L, Gomez RS. Novel mutations in the SH3BP2 gene associated with sporadic central giant cell lesions and cherubism. Oral Dis. 2009;15(1):106-10.

14. Lo B, Faiyaz - Ul - Haque M, Kennedy S, Aviv R, Tsui LC, Teebi AS. Novel mutation in the gene encoding $\mathrm{c}-\mathrm{Abl}$ - binding protein SH3BP2 causes cherubism. Am J Med Gen. 2003;121(1):37-40.

15. Mukai T, Gallant R, Ishida S, Kittaka M, Yoshitaka T, Fox DA, Morita Y, Nishida K, Rottapel R, Ueki Y. Loss of SH3 Domain - Binding Protein 2 Function Suppresses Bone Destruction in Tumor Necrosis Factor - Driven and Collage-Induced Arthritis in Mice. Arthritis Rheumatol. 2015;67(3):656-67.

16. Mukai T, Gallant R, Ishida S, Yoshitaka T, Kittaka M, Nishida K, Fox DA, Morita Y, Ueki Y. SH3BP2 gain-of-function mutation exacerbates inflammation and bone loss in a murine collagen-induced arthritis model. PloS one. 2014;9(8):e105518.

17. Lodish H, Berk A, Zipursky SL, Matsudaira P, Baltimore D, Darnell J. Molecular cell biology 4th edition. National Center for Biotechnology Information, Bookshelf. 2000 Jan 1.

18. Cooper DN, Chen JM, Ball EV, Howells K, Mort M, Phillips AD, Chuzhanova N, Krawczak M, Kehrer - Sawatzki H, Stenson PD. Genes, mutations, and human inherited disease at the dawn of the age of personalized genomics. Hum Mut. 2010 Jun 1;31(6):631-55

19. Tartaglia M, Kalidas K, Shaw A, Song X, Musat DL, van der Burgt I, Brunner HG, Bertola DR, Crosby A, Ion A, Kucherlapati RS. PTPN11 mutations in Noonan syndrome: molecular spectrum, genotype-phenotype correlation, and phenotypic heterogeneity. Am J Hum Gen. 2002;70(6):1555-63.

20. Tartaglia M, Zampino GI, Gelb BD. Noonan syndrome: clinical aspects and molecular pathogenesis. Molecular syndromology. 2010;1(1):2-6.

21. Carvalho VM, Perdigao PF, Amaral FR, De Souza PE, De Marco L, Gomez RS. Novel mutations in the SH3BP2 gene associated with sporadic central giant cell lesions and cherubism. Oral Dis. 2009;15(1):106-10.

22. Tchurikov NA. Molecular mechanisms of epigenetics. Biochemistry. 2005;70(4):40623.

23. Epigenetic Modifications Regulate Gene Expression. 2008.

24. Handy DE, Castro R, Loscalzo J. Epigenetic modifications: basic mechanisms and role in cardiovascular disease. Circulation. 2011;123(19):2145-56.

25. Park-Min KH, Lim E, Lee MJ, Park SH, Giannopoulou E, Yarilina A, Van Der Meulen M, Zhao B, Smithers N, Witherington J, Lee K. Inhibition of osteoclastogenesis and inflammatory bone resorption by targeting BET proteins and epigenetic regulation. $\mathrm{Na}-$ ture communications. 2014;5:5418. 